\title{
Existence of Two Periodic Solutions to General Anisotropic Euler-Lagrange Equations
}

\section{Magdalena Chmara}

Abstract. This paper is concerned with the following Euler-Lagrange system

$$
\left\{\begin{array}{l}
\frac{d}{d t} \mathcal{L}_{v}(t, u(t), \dot{u}(t))=\mathcal{L}_{x}(t, u(t), \dot{u}(t)) \quad \text { for a.e. } t \in[-T, T] \\
u(-T)=u(T) \\
\mathcal{L}_{v}(-T, u(-T), \dot{u}(-T))=\mathcal{L}_{v}(T, u(T), \dot{u}(T))
\end{array}\right.
$$

where Lagrangian is given by $\mathcal{L}=F(t, x, v)+V(t, x)+\langle f(t), x\rangle$, growth conditions are determined by an anisotropic G-function and some geometric conditions at infinity. We consider two cases: with and without forcing term $f$. Using a general version of the mountain pass theorem and Ekeland's variational principle we prove the existence of at least two nontrivial periodic solutions in an anisotropic Orlicz-Sobolev space.

\section{Introduction}

We consider the second order system

$$
\left\{\begin{array}{l}
\frac{d}{d t} \mathcal{L}_{v}(t, u(t), \dot{u}(t))=\mathcal{L}_{x}(t, u(t), \dot{u}(t)) \quad \text { for a.e. } t \in I, \\
u(-T)=u(T), \\
\mathcal{L}_{v}(-T, u(-T), \dot{u}(-T))=\mathcal{L}_{v}(T, u(T), \dot{u}(T)),
\end{array}\right.
$$

where $I=[-T, T],|I| \geq 1$ and $\mathcal{L}: I \times \mathbb{R}^{N} \times \mathbb{R}^{N} \rightarrow \mathbb{R}$ is given by

$$
\mathcal{L}(t, x, v)=F(t, x, v)+V(t, x)+\langle f(t), x\rangle .
$$

If $\mathcal{L}_{v}$ is strictly convex with respect to $v$, then $\mathcal{L}_{v}(-T, u(-T), \dot{u}(-T))=\mathcal{L}_{v}(T, u(T), \dot{u}(T))$ is equivalent to $\dot{u}(-T)=\dot{u}(T)$.

The growth of $\mathcal{L}$ is determined by function $G$ such that

(G) $G: \mathbb{R}^{N} \rightarrow[0, \infty)$ is a continuously differentiable G-function (i.e., $G$ is convex, even, $G(0)=0$ and $G(x) /|x| \rightarrow \infty$ as $|x| \rightarrow \infty$ ) satisfying $\Delta_{2}$ and $\nabla_{2}$ conditions (at infinity).

Received March 12, 2020; Accepted September 9, 2020.

Communicated by Eiji Yanagida.

2010 Mathematics Subject Classification. 46E30, 46E40.

Key words and phrases. anisotropic Orlicz-Sobolev space, Euler-Lagrange equations, mountain pass theorem, Palais-Smale condition. 
In Section 2 we use $G$ to define an Orlicz space. The following assumption on $F, V$ and $f$ will be needed throughout the paper.

Function $F: I \times \mathbb{R}^{N} \times \mathbb{R}^{N} \rightarrow \mathbb{R}$ is of class $C^{1}$ and satisfy

$\left(\mathrm{F}_{1}\right) F(t, x, \cdot)$ is convex for all $(t, x) \in I \times \mathbb{R}^{N}$.

$\left(\mathrm{F}_{2}\right)$ there exist $a \in C\left(\mathbb{R}_{+}, \mathbb{R}_{+}\right)$and $b \in \mathbf{L}^{1}\left(I, \mathbb{R}_{+}\right)$such that for all $(t, x, v) \in I \times \mathbb{R}^{N} \times \mathbb{R}^{N}$,

$$
\begin{aligned}
|F(t, x, v)| & \leq a(|x|)(b(t)+G(v)), \\
\left|F_{x}(t, x, v)\right| & \leq a(|x|)(b(t)+G(v)), \\
G^{*}\left(F_{v}(t, x, v)\right) & \leq a(|x|)(b(t)+G(v)) .
\end{aligned}
$$

$\left(\mathrm{F}_{3}\right)$ there exists $\theta_{F}>0$ such that for all $(t, x, v) \in I \times \mathbb{R}^{N} \times \mathbb{R}^{N}$,

$$
\left\langle F_{x}(t, x, v), x\right\rangle+\left\langle F_{v}(t, x, v), v\right\rangle \leq \theta_{F} F(t, x, v) .
$$

$\left(\mathrm{F}_{4}\right)$ there exists $\Lambda>0$ such that for all $(t, x, v) \in I \times \mathbb{R}^{N} \times \mathbb{R}^{N}$,

$$
F(t, x, v) \geq \Lambda G(v)
$$

$\left(\mathrm{F}_{5}\right) F(t, x, 0)=0, F_{v}(t, x, 0)=0$ for all $(t, x) \in I \times \mathbb{R}^{N}$.

On potential $V \in C^{1}\left(I \times \mathbb{R}^{N}, \mathbb{R}\right)$ we assume

$\left(\mathrm{V}_{1}\right) V(t, x)=K(t, x)-W(t, x)$ for $x \in \mathbb{R}^{N}, t \in I$,

$\left(\mathrm{V}_{2}\right)$ there exist $M>0, \varepsilon_{V}>0, \theta_{V}>\theta_{F}, 1<p_{K} \leq \theta_{V}-\varepsilon_{V}$, such that

$$
\left\langle V_{x}(t, x), x\right\rangle \leq\left(\theta_{V}-\varepsilon_{V}\right) K(t, x)-\theta_{V} W(t, x)
$$

and

$$
W(t, x)>K(t, x)>|x|^{p_{K}}
$$

for $|x|>M, t \in I$,

$\left(\mathrm{V}_{3}\right)$ there exist $\rho>0, b>1$ and $g \in \mathbf{L}^{1}(I, \mathbb{R})$, such that $V(t, x) \geq b G(x)-g(t)$ for $G(x /(2|I|)) \leq \rho / 2$

$\left(\mathrm{V}_{4}\right) \int_{I} V(t, 0) d t=0$ for $t \in I$.

We assume that the forcing term $f$ belongs to the space $\mathbf{L}^{G^{\star}}$ and

(f) $\int_{I} G^{*}(f(t))+g(t) d t<\min \{\Lambda, b-1\} \rho$. 
Using the general form of the mountain pass theorem and Ekeland's variational principle we show that the problem (1.1) has at least two nontrivial solutions in an anisotropic Orlicz-Sobolev space (see Theorems 3.1, 4.2 and 4.4).

Problems similar to (1.1) were considered e.g. in [9,20,22] for $F(t, x, v)=|v|^{p}$, in [1] for $F$ being an isotropic G-function and in 7,17 (periodic problem) for $F$ being an anisotropic G-function. Recently in [8] authors proved the existence of a Dirichlet problem, where $F$ is in general form and satisfies $\left(\mathrm{F}_{1}\right)-\left(\mathrm{F}_{5}\right)$.

In $7,9,20,22$ the existence of a mountain pass type solution was shown using the well known mountain pass theorem by Ambrosetti and Rabinowitz [2]. One of the assumptions of this theorem is that the action functional is greater than zero on the boundary of some ball, i.e.,

$$
\text { there exists } r>0 \text { such that if }\|x\|=r \text {, then } J(x)>0 \text {. }
$$

In the Orlicz-Sobolev space norm is a sum of Luxemburg norms $\|u\|_{\mathbf{W}^{1} \mathbf{L}^{G}}=\|\dot{u}\|_{\mathbf{L}^{G}}+\|u\|_{\mathbf{L}^{G}}$ (see more in Section 2). To apply theorem of Ambrosetti and Rabinowitz to the anisotropic Orlicz-Sobolev space setting it was necessary to establish connections between Luxemburg norm $\|\cdot\|_{\mathbf{L}^{G}}$ and modular $\int_{I} G(\cdot) d t$ (see papers $\left.4,7,8\right]$ ).

It turns out that the ball $B_{r}=\left\{u \in \mathbf{W}^{1} \mathbf{L}^{G}:\|u\|_{\mathbf{W}^{1} \mathbf{L}^{G}}<r\right\}$ is not the most suitable set to obtain the mountain pass geometry in the anisotropic case (an example explaining this fact can be found in Section 5). Therefore, instead of the ball, we use the set

$$
\Omega=\Phi^{-1}([0, \rho))=\left\{u \in \mathbf{W}^{1} \mathbf{L}^{G}: \int_{I} G(\dot{u})+G(u) d t<\rho\right\},
$$

where $\Phi: \mathbf{W}^{1} \mathbf{L}^{G} \rightarrow[0, \infty)$ is given by $\Phi(u)=\int_{I} G(\dot{u})+G(u) d t$. $\Phi$ is not a norm, but it is better suited to geometric idea of MPT in the anisotropic case.

In the literature we can find a lot of versions of the mountain pass theorem. In our case we use general form of the MPT with a bounded open neighborhood instead of the ball. The following theorem is a direct consequence of Theorem 4.10 in [16].

Theorem 1.1. Let $X$ be a Banach space and $J \in C^{1}(X, \mathbb{R})$. Assume that there exist $e_{0}, e_{1} \in X$ and a bounded open neighborhood $\Omega$ of $e_{0}$ such that $e_{1} \in X \backslash \Omega$ and

$$
\inf _{u \in \partial \Omega} J(u)>\max \left\{J\left(e_{0}\right), J\left(e_{1}\right)\right\} .
$$

Let

$$
\Gamma=\left\{g \in C([0,1], X): g(0)=e_{0}, g(1)=e_{1}\right\} \quad \text { and } \quad c=\inf _{g \in \Gamma \in[0,1]} \max _{s \in[(g(s)) .}
$$

If $J$ satisfies the Palais-Smale condition, then $c$ is a critical value of $J$ and

$$
c>\max \left\{J\left(e_{0}\right), J\left(e_{1}\right)\right\} .
$$


In fact, there is only one difference between Theorem 1.1 and Theorem 4.10 in [16]. In [16] it is assumed that $J$ satisfies the Palais Smale condition at the level $c$. When the Palais-Smale condition is satisfied (i.e., $\left\{J\left(u_{n}\right)\right\}$ is bounded and $J^{\prime}\left(u_{n}\right) \rightarrow 0$ ), we can check immediately that the Palais Smale condition at the level $c$ (i.e., $\left\{J\left(u_{n}\right)\right\} \rightarrow c$ and $J^{\prime}\left(u_{n}\right) \rightarrow 0$ ) holds for all $c \in \mathbb{R}[12$, p. 16].

In the proof of Theorem 3.1 we show that our action functional satisfies conditions of Theorem 1.1 where $\Omega$ is given by (1.4).

Using the Ekeland Variational Principle we show the existence of a second nontrivial solution of (1.1), which belongs to the interior of $\Omega$. The existence of two solutions was investigated for example in 22] for ordinary $p$-Laplacian systems and in [21] for $p(t)$ Laplacian systems. In these papers it was shown that there exists $u_{2}$ such that

$$
\mathcal{J}\left(u_{2}\right)=\inf _{u \in \overline{B_{r}}} \mathcal{J}(u) \leq 0 \quad(\text { or }<0, \text { in } 22)
$$

and that there exists a minimizing sequence $\left\{v_{n}\right\}$ which is a Palais-Smale sequence of $\mathcal{J}$, contained in a small ball centered at 0 . In our case we use similar methods, but instead of the ball $B_{r}$ we take $\Omega=\Phi^{-1}([0, \rho))$. Since $\Omega$ is not a ball we cannot simply cite 21,22 . Our proof is based on concepts of 3,16$]$.

We shall distinguish cases with and without forcing. In the case with forcing it is enough that

$$
\inf _{u \in \bar{\Omega}} \mathcal{J}(u) \leq 0 .
$$

In the case without forcing $u_{0} \equiv 0$ is a trivial solution of 1.1 , so we need the sharp inequality. To obtain this we need additional assumptions:

$\left(\mathrm{F}_{6}\right)$ there exist $\zeta_{F}>1, \lambda_{0} \in(0,1)$ such that

$$
F(t, \lambda x, \lambda v) \leq \lambda^{\zeta_{F}} F(t, x, v)
$$

for $\lambda \in\left(0, \lambda_{0}\right), t \in I, G\left(\frac{x}{2|I|}\right) \leq \rho / 2$,

$\left(\mathrm{V}_{5}\right)$ there exist $\zeta_{K}, \zeta_{W}>1, \zeta_{W}<\min \left\{\zeta_{F}, \zeta_{K}\right\}, \lambda_{0} \in(0,1)$ and $\bar{W}: I \times \mathbb{R}^{N} \rightarrow(0, \infty)$ such that

$$
V(t, \lambda x) \leq \lambda^{\zeta_{K}} K(t, x)-\lambda^{\zeta_{W}} \bar{W}(t, x)
$$

for $\lambda \in\left(0, \lambda_{0}\right), t \in I, G\left(\frac{x}{2|I|}\right) \leq \rho / 2$.

\section{Preliminaries}

A function $G^{*}(y)=\sup _{x \in \mathbb{R}^{N}}\{\langle x, y\rangle-G(x)\}$ is called the Fenchel conjugate of $G$. As an immediate consequence of the definition we have the Fenchel-Young inequality

$$
\forall x, y \in \mathbb{R}^{N}, \quad\langle x, y\rangle \leq G(x)+G^{*}(y) .
$$


Now we briefly recall the notion of anisotropic Orlicz spaces. For more details we refer the reader to 6,19$]$. The Orlicz space associated with $G$ is defined to be

$$
\mathbf{L}^{G}=\mathbf{L}^{G}\left(I, \mathbb{R}^{N}\right)=\left\{u: I \rightarrow \mathbb{R}^{N}: \int_{I} G(u) d t<\infty\right\} .
$$

The space $\mathbf{L}^{G}$ equipped with the Luxemburg norm

$$
\|u\|_{\mathbf{L}^{G}}=\inf \left\{\lambda>0: \int_{I} G\left(\frac{u}{\lambda}\right) d t \leq 1\right\}
$$

is a reflexive Banach space. We have the Hölder inequality

$$
\int_{I}\langle u, v\rangle d t \leq 2\|u\|_{\mathbf{L}^{G}}\|v\|_{\mathbf{L}^{G^{*}}} \quad \text { for every } u \in \mathbf{L}^{G} \text { and } v \in \mathbf{L}^{G^{*}} .
$$

Let us denote by

$$
\mathbf{W}^{1} \mathbf{L}^{G}=\mathbf{W}^{1} \mathbf{L}^{G}\left(I, \mathbb{R}^{N}\right)=\left\{u \in \mathbf{L}^{G}: \dot{u} \in \mathbf{L}^{G}\right\}
$$

an anisotropic Orlicz-Sobolev space of vector valued functions with the usual norm

$$
\|u\|_{\mathbf{W}^{1} \mathbf{L}^{G}}=\|u\|_{\mathbf{L}^{G}}+\|\dot{u}\|_{\mathbf{L}^{G}} .
$$

It is known that elements of $\mathbf{W}^{1} \mathbf{L}^{G}$ are absolutely continuous functions.

We introduce the following subset of $\mathbf{W}^{1} \mathbf{L}^{G}$

$$
\mathbf{W}_{T}^{1} \mathbf{L}^{G}=\left\{u \in \mathbf{W}^{1} \mathbf{L}^{G}: u(-T)=u(T)\right\} .
$$

Functional $R_{G}: \mathbf{L}^{G} \rightarrow[0, \infty)$ given by formula $R_{G}(u):=\int_{I} G(u) d t$ is called modular (see 11, 14, 18). For Lebesgue spaces notions of modular and norm are indistinguishable because

$$
\|u\|_{\mathbf{L}^{p}}=\left(\int_{I}|u(t)|^{p} d t\right)^{1 / p} .
$$

In the Orlicz space this relation is more complicated. One can easily see, that

$$
R_{G}(u) \leq\|u\|_{\mathbf{L}^{G}} \text { for }\|u\|_{\mathbf{L}^{G}} \leq 1 \quad \text { and } \quad R_{G}(u)>\|u\|_{\mathbf{L}^{G}} \text { for }\|u\|_{\mathbf{L}^{G}}>1
$$

The modular $R_{G}$ is coercive in the following sense [15, Proposition 2.7]:

$$
\lim _{\|u\|_{\mathbf{L}} \rightarrow \infty} \frac{R_{G}(u)}{\|u\|_{\mathbf{L}^{G}}}=\infty .
$$

The following lemma is crucial to Theorems 3.1 and 4.2 . We will use it to show that $\left.\mathcal{J}\right|_{\partial \Omega}>0$ and that $\mathcal{J}$ is negative in the interior of $\Omega$.

Lemma 2.1. If $|I| \leq 1$, then $R_{G}(\dot{u})+R_{G}(u) \geq 2|I| G\left(\frac{u(t)}{2}\right)$ for $t \in I, u \in \mathbf{W}^{1} \mathbf{L}^{G}$. If $|I| \geq 1$ then $R_{G}(\dot{u})+R_{G}(u) \geq 2 G\left(\frac{u(t)}{2|I|}\right)$ for $t \in I, u \in \mathbf{W}^{1} \mathbf{L}^{G}$. 
Proof. Let $u \in \mathbf{W}^{1} \mathbf{L}^{G}$. Since $u$ is absolutely continuous we have

$$
u(t)-u(s)=\int_{s}^{t} \dot{u} d t \quad \text { for } s, t \in I .
$$

Hence, by Jensen's integral inequality and since $G(k x) / k$ is increasing with respect to $k>0$ for $x \in \mathbb{R}^{N}$ we obtain

$$
\begin{aligned}
& G\left(\frac{1}{|I|}\left(u(t)-\frac{1}{|I|} \int_{I} u(s) d s\right)\right)=G\left(\frac{1}{|I|}\left(\int_{I} \frac{u(t)-u(s)}{|I|} d s\right)\right) \\
\leq & \frac{1}{|I|} \int_{I} G\left(\frac{u(t)-u(s)}{|I|}\right) d s=\frac{1}{|I|} \int_{I} G\left(\frac{1}{|I|} \int_{s}^{t} \dot{u}(r) d r\right) d s \\
= & \frac{1}{|I|} \int_{I} G\left(\frac{1}{|t-s|} \int_{s}^{t} \frac{|t-s|}{|I|} \dot{u}(r) d r\right) d s \leq \frac{1}{|I|} \int_{I} \frac{1}{|t-s|} \int_{s}^{t} G\left(|t-s| \frac{\dot{u}(r)}{|I|}\right) d r d s \\
\leq & \frac{1}{|I|} \int_{I} \frac{1}{|I|} \int_{s}^{t} G(\dot{u}(r)) d r d s \leq \frac{1}{|I|} \int_{I} G(\dot{u}(r)) d r .
\end{aligned}
$$

Let $|I| \leq 1$. Then by 2.2 , Jensen's integral inequality and convexity of $G$ we have that for all $t \in I$,

$$
\int_{I} G(u)+G(\dot{u}) d t \geq|I|\left(G\left(\frac{1}{|I|} \int_{I} u\right)+G\left(\frac{1}{|I|}\left(u(t)-\frac{1}{|I|} \int_{I} u\right)\right)\right) \geq 2|I| G\left(\frac{u(t)}{2}\right) .
$$

Let $|I| \geq 1$. Then by $(2.2)$, Jensen's integral inequality and convexity of $G$ we have that for all $t \in I$,

$$
\begin{aligned}
\int_{I} G(u)+G(\dot{u}) d t & \geq\left(G\left(\frac{1}{|I|^{2}} \int_{I} u\right)+G\left(\frac{1}{|I|} u(t)-\frac{1}{|I|^{2}} \int_{I} u\right)\right) \\
& \geq 2 G\left(\frac{u(t)}{2|I|}\right) .
\end{aligned}
$$

In the proof of the existence of a second solution we will need the following inequality by Brezis and Lieb [5, Lemma 3] (see also [14, Lemma 4.7]).

Lemma 2.2. For all $k>1,0<\epsilon<1 / k, x, y \in \mathbb{R}^{N}$, we have

$$
|G(x+y)-G(x)| \leq \epsilon|G(k x)-k G(x)|+2 G\left(C_{\epsilon} y\right),
$$

where $C_{\epsilon}=\frac{1}{\epsilon(k-1)}$.

Let us also recall Proposition 2.4 in [7], which will be used in the proof of the fact that our action functional satisfies the Palais-Smale condition.

Proposition 2.3. For any $1<p \leq q<\infty$, such that $|\cdot|^{p} \prec G(\cdot) \prec|\cdot|^{q}$,

$$
\int_{I}|u|^{p} d t \geq C\|u\|_{\mathbf{W}^{1} \mathbf{L}^{G}}^{p-q}\|u\|_{\mathbf{L}^{G}}^{q}
$$

for $u \in \mathbf{W}^{1} \mathbf{L}^{G} \backslash\{0\}$ and some $C>0$. 


\section{Existence of the first solution}

Define the action functional $\mathcal{J}: \mathbf{W}_{T}^{1} \mathbf{L}^{G}\left(I, \mathbb{R}^{N}\right) \rightarrow \mathbb{R}$ by

$$
\mathcal{J}(u)=\int_{I} F(t, u, \dot{u})+V(t, u)+\langle f, u\rangle d t
$$

Since $F$ and $V$ are of class $C^{1}$ and $F$ satisfies $\left(\mathrm{F}_{2}\right), \mathcal{J}$ is well defined and of class $C^{1}$. Furthermore, its derivative is given by

$$
\mathcal{J}^{\prime}(u) \varphi=\int_{I}\left\langle F_{v}(t, u, \dot{u}), \dot{\varphi}\right\rangle d t+\int_{I}\left\langle F_{x}(t, u, \dot{u})+V_{x}(t, u)+f, \varphi\right\rangle d t .
$$

For more details see [6, Theorem 5.7].

We can now formulate the first of our main results.

Theorem 3.1. Let $F, V$ and $f$ satisfies $\left(\mathrm{F}_{1}\right)-\left(\mathrm{F}_{5}\right),\left(\mathrm{V}_{1}\right)-\left(\mathrm{V}_{4}\right)$ and $(\mathrm{f})$. Then (1.1) has at least one nontrivial periodic solution.

Proof. We show that $\mathcal{J}$ satisfies assumptions of Theorem 1.1 for $\Omega=\Phi^{-1}([0, \rho))$, where $\rho>0, \Phi: \mathbf{W}^{1} \mathbf{L}^{G} \rightarrow[0, \infty), \Phi(u)=R_{G}(\dot{u})+R_{G}(u)$.

Step 1. One can see, that $\Omega$ is a bounded open neighborhood of 0 . We claim that $\partial \Omega=\Phi^{-1}(\{\rho\})$. Since $\Phi$ is continuous,

$$
\partial \Phi^{-1}([0, \rho)) \subset \Phi^{-1}(\partial[0, \rho))=\Phi^{-1}(\{\rho\})
$$

For the opposite inclusion (not true in general) suppose that $x \in \Phi^{-1}(\{\rho\}), x_{n}^{1}=\frac{n+1}{n} x$, $x_{n}^{2}=\frac{n}{n+1} x$ for $n \in \mathbb{N}$. Then $x_{n}^{1}, x_{n}^{2} \rightarrow x$. From convexity of $\Phi$ and since $\rho>0$, we have

$$
\Phi\left(x_{n}^{1}\right) \geq \frac{n+1}{n} \Phi(x)>\Phi(x) \quad \text { and } \quad \Phi\left(x_{n}^{2}\right) \leq \frac{n}{n+1} \Phi(x)<\Phi(x)
$$

for all $n \in \mathbb{N}$. Hence $x \in \partial \Omega$.

Step 2. In this step we show that $\mathcal{J}$ satisfies the Palais-Smale condition. Fix $u \in$ $\mathbf{W}_{T}^{1} \mathbf{L}^{G}$, such that $\|u\|_{\mathbf{W}^{1} \mathbf{L}^{G}} \neq 0$. From $\left(\mathrm{F}_{3}\right)$ we have

$$
\int_{I}\left\langle F_{v}(t, u, \dot{u}), \dot{u}\right\rangle+\left\langle F_{x}(t, u, \dot{u}), u\right\rangle d t \leq \theta_{F} \int_{I} F(t, u, \dot{u}) d t .
$$

Combining it with $\left(\mathrm{F}_{4}\right)$ we have

$$
\int_{I} \theta_{V} F(t, u, \dot{u})-\left\langle F_{v}(t, u, \dot{u}), \dot{u}\right\rangle-\left\langle F_{x}(t, u, \dot{u}), u\right\rangle d t \geq \Lambda\left(\theta_{V}-\theta_{F}\right) \int_{I} G(\dot{u}) d t .
$$

Set

$$
M_{V}=\sup \left\{\left|\left(\theta_{V}-\varepsilon_{V}\right) K(t, x)-\theta_{V} W(t, x)-\left\langle V_{x}(t, x), x\right\rangle\right|: t \in I,|x| \leq M\right\}
$$


Then, by Assumptions $\left(\mathrm{V}_{1}\right)$ and $\left(\mathrm{V}_{2}\right)$,

$$
\begin{aligned}
& \theta_{V} \int_{I} V(t, u)-\left\langle V_{x}(t, u), u\right\rangle d t \\
= & \varepsilon_{V} \int_{I} K(t, u) d t+\left(\theta_{V}-\varepsilon_{V}\right) \int_{I} K(t, u) d t-\theta_{V} \int_{I} W(t, u) d t-\int_{I}\left\langle V_{x}(t, u), u\right\rangle d t \\
\geq & \varepsilon_{V} \int_{I} K(t, u) d t-|I| M_{V} .
\end{aligned}
$$

Applying 1.2 and Proposition 2.3 we have that for some $C_{1}, C_{2}=C_{2}(|I|)>0$,

$$
\int_{I} K(t, u) d t \geq C_{1} \frac{\|u\|_{\mathbf{L}^{G}}^{q}}{\|u\|_{\mathbf{W}^{1} \mathbf{L}^{G}}^{q-p_{K}}}-C_{2}
$$

for all $q$ such that $G \prec|\cdot|^{q}$.

Since $f \in \mathbf{L}^{G^{*}}$, by Hölder's inequality we have

$$
\left(\theta_{V}-1\right) \int_{I}\langle f(t), u\rangle d t \geq-2\left(\theta_{V}-1\right)\|f\|_{\mathbf{L}^{G^{*}}}\|u\|_{\mathbf{L}^{G}} \geq-C_{f}\|u\|_{\mathbf{W}^{1} \mathbf{L}^{G}},
$$

where $C_{f}=2\left(\theta_{V}-1\right)\|f\|_{\mathbf{L}^{G^{\star}}}>0$.

Let $\left\{u_{n}\right\} \subset \mathbf{W}^{1} \mathbf{L}^{G}$ be a Palais-Smale sequence for functional $\mathcal{J}$. Then there exist $C_{J}, C_{J^{\prime}}>0$ such that

$$
-C_{J} \leq \mathcal{J}\left(u_{n}\right) \leq C_{J}, \quad-C_{J^{\prime}}\left\|u_{n}\right\|_{\mathbf{W}^{1} \mathbf{L}^{G}} \leq \mathcal{J}^{\prime}\left(u_{n}\right) u_{n} \leq C_{J^{\prime}}\left\|u_{n}\right\|_{\mathbf{W}^{1} \mathbf{L}^{G}} .
$$

Assume that $\left\{u_{n}\right\}$ is not bounded. Then there exists a subsequence of $\left\{u_{n}\right\}$ such that $\left\|u_{n}\right\|_{\mathbf{W}^{1} \mathbf{L}^{G}} \rightarrow \infty$.

Combining (3.2)-3.6), we obtain

$$
\begin{aligned}
& \theta_{V} C_{J}+C_{J^{\prime}}\left\|u_{n}\right\|_{\mathbf{W}^{1} \mathbf{L}^{G}} \geq \theta_{V} \mathcal{J}\left(u_{n}\right)-\mathcal{J}^{\prime}\left(u_{n}\right) u_{n} \\
= & \int_{I} \theta_{V} F\left(t, u_{n}, \dot{u}_{n}\right)-\left\langle F_{v}\left(t, u_{n}, \dot{u}_{n}\right), \dot{u}_{n}\right\rangle-\left\langle F_{x}\left(t, u_{n}, \dot{u}_{n}\right), u_{n}\right\rangle d t \\
& +\int_{I} \theta_{V} V\left(t, u_{n}\right)-\left\langle V_{x}\left(t, u_{n}\right), u_{n}\right\rangle d t+\int_{I}\left(\theta_{V}-1\right)\left\langle f(t), u_{n}\right\rangle d t \\
\geq & C_{0} \int_{I} G\left(\dot{u}_{n}\right) d t+C_{1} \frac{\left\|u_{n}\right\|_{\mathbf{L}^{G}}^{q}}{\left\|u_{n}\right\|_{\mathbf{W}^{1} \mathbf{L}^{G}}^{q-p_{K}}}-C_{2}-C_{3}\left\|u_{n}\right\|_{\mathbf{W}^{1} \mathbf{L}^{G}}
\end{aligned}
$$

for some $C_{0}, C_{1}, C_{2}, C_{3}>0$. Hence

$$
\left\|u_{n}\right\|_{\mathbf{W}^{1} \mathbf{L}^{G}}\left(\frac{R_{G}\left(\dot{u}_{n}\right)}{\left\|u_{n}\right\|_{\mathbf{W}^{1} \mathbf{L}^{G}}}+\frac{\left\|u_{n}\right\|_{\mathbf{L}^{G}}^{q}}{\left\|u_{n}\right\|_{\mathbf{W}^{1} \mathbf{L}^{G}}^{q-p_{K}+1}}-\frac{C_{4}}{\left\|u_{n}\right\|_{\mathbf{W}^{1} \mathbf{L}^{G}}}-C_{5}\right) \leq C_{6}
$$

for some $C_{4}, C_{5}, C_{6}>0$.

In the proof of Lemma 3.2 in $[7]$ it was shown that the left-hand side of the above inequality goes to the infinity, which is impossible. Hence $\left\{u_{n}\right\}$ is bounded. 
Repeating arguments used in the proof of Lemma 4.2 in [8 one can show that there exists a converging subsequence, i.e., the Palais Smale condition is satisfied.

Step 3. Take $u \in \Phi^{-1}(\{\rho\})$. Then, by Lemma 2.1, we have $G\left(\frac{u(t)}{2|I|}\right) \leq \rho / 2$ for all $t \in I$. From $\left(\mathrm{F}_{4}\right),\left(\mathrm{V}_{3}\right)$ and Fenchel's inequality,

$$
\begin{aligned}
\mathcal{J}(u) & \geq \int_{I} \Lambda G(\dot{u})+b G(u)-G(u)-G^{*}(f)-g(t) d t \\
& \geq \min \{\Lambda, b-1\} \rho-\int_{I}\left(G^{*}(f)+g(t)\right) d t .
\end{aligned}
$$

Combining it with (f) we obtain $\mathcal{J}(u)>0$ on $\Phi^{-1}(\{\rho\})$.

Step 4. Now we show that there exists $e \in \Phi^{-1}((\rho, \infty))$ such that $\mathcal{J}(e)<0$. By $\left(\mathrm{V}_{2}\right)$, for $|x|>M, t \in I, \lambda>1$ we obtain

$$
\begin{aligned}
\log \left(\frac{-V(t, \lambda x)}{-V(t, x)}\right) & =\int_{1}^{\lambda} \frac{d}{d \lambda} \log (-V(t, \lambda x)) d \lambda=\int_{1}^{\lambda} \frac{-\left\langle V_{x}(t, \lambda x), \lambda x\right\rangle}{-\lambda V(t, \lambda x)} d \lambda \\
& \geq \int_{1}^{\lambda} \frac{-\left(\theta_{V}-\varepsilon_{V}\right) K(t, \lambda x)+\theta_{V} W(t, \lambda x)}{-\lambda V(t, \lambda x)} d \lambda \geq \theta_{V} \int_{1}^{\lambda} \frac{1}{\lambda}=\log \left(\lambda^{\theta_{V}}\right) .
\end{aligned}
$$

Hence

$$
V(t, \lambda x) \leq \lambda^{\theta_{V}} V(t, x) \text { for }|x|>M
$$

In similar way, from $\left(\mathrm{F}_{3}\right)$, we have

$$
F(t, \lambda x, \lambda v) \leq \lambda^{\theta_{F}} F(t, x, v)
$$

for $x, v \in \mathbb{R}^{N}, t \in I, \lambda>1$. Let $\lambda>1$ and $\psi \in \mathbf{W}_{T}^{1} \mathbf{L}^{G}$ be such that $\mid\{t \in I:|\psi(t)|>$ $0\} \mid>0$. Then we obtain

$$
\mathcal{J}(\lambda \psi) \leq \int_{I} \lambda^{\theta_{F}} F(t, \psi, \dot{\psi})+\lambda\langle f(t), \psi\rangle d t+\int_{\{|\psi(t)|>M\}} \lambda^{\theta_{V}} V(t, \psi) d t+C_{V}|I|
$$

where $C_{V}=\sup \{V(t, x):|x|<M, t \in I\}$. Note that $V$ is negative for $|x|>M$ and $\theta_{V}>\theta_{F}$. Therefore, if we take $e=\lambda \psi$ for sufficiently large $\lambda$, we get $\mathcal{J}(e)<0$ and $\Phi(e)>\rho$.

Step 5. To finish the proof note that by (3.1), $\left(\mathrm{F}_{5}\right)$ and $\left(\mathrm{V}_{4}\right)$ we have that $\mathcal{J}(0)=0$. Applying Theorem 1.1 to $\mathcal{J}, e_{0}=0$ and $e_{1}=e$, we obtain that there exists a critical point $u_{1}$ with a critical value

$$
c_{1}:=\inf _{g \in \Gamma} \max _{s \in[0,1]} \mathcal{J}(g(s))>0
$$

where

$$
\Gamma=\left\{g \in C\left([0,1], \mathbf{W}^{1} \mathbf{L}^{G}\right) \mid g(0)=0, g(1)=e\right\}
$$




\section{Existence of the second solution}

Theorem 3.1 ensures the existence of the first solution of (1.1). To obtain the second solution we use the well known Ekeland's Variational Principle.

Theorem 4.1. [10, Theorem 1.1] Let $V$ be a complete metric space and $J: V \rightarrow \mathbb{R} \cup\{+\infty\}$ a lower semi continuous function, bounded from below, $\not \equiv+\infty$. Let $u \in V$ and $\varepsilon>0$ be such that

$$
J(u) \leq \inf _{v \in V} J(v)+\varepsilon .
$$

Then for all $\delta>0$ there exists some point $v \in V$ such that

(i) $J(v) \leq J(u)$,

(ii) $d(u, v) \leq \delta$,

(iii) $J(w)>J(v)-\frac{\varepsilon}{\delta} d(v, w)$ for all $w \neq v$.

Set

$$
c_{2}:=\inf _{u \in \bar{\Omega}} \mathcal{J}(u)
$$

Let us recall, that $\Omega=\Phi^{-1}([0, \rho))$. Firstly we consider the case with forcing.

Theorem 4.2. Let $F$ and $V$ satisfies $\left(\mathrm{F}_{1}\right)-\left(\mathrm{F}_{5}\right),\left(\mathrm{V}_{1}\right)-\left(\mathrm{V}_{4}\right)$ and $f(t) \not \equiv 0$. Then (1.1) has at least two periodic solutions.

Proof. Note that $\bar{\Omega}$ is a complete metric space with respect to the norm in $\mathbf{W}^{1} \mathbf{L}^{G}$ and $\mathcal{J}$ is bounded from below on $\bar{\Omega}$. Fix $\varepsilon>0$ and choose $\delta=\sqrt{\varepsilon}$. There exists $u \in \bar{\Omega}$ such that $\mathcal{J}(u) \leq c_{2}+\varepsilon$. By Theorem 4.1. there exists $v \in \bar{\Omega}$ such that

$$
\begin{gathered}
c_{2} \leq \mathcal{J}(v) \leq c_{2}+\varepsilon \\
\|v-u\|_{\mathbf{W}^{1} \mathbf{L}^{G}} \leq \sqrt{\varepsilon} \\
\mathcal{J}(w) \geq \mathcal{J}(v)-\sqrt{\varepsilon}\|w-v\|_{\mathbf{W}^{1} \mathbf{L}^{G}} \quad \text { for all } w \neq v .
\end{gathered}
$$

Now we show that $v \in \Omega$. Since $\mathcal{J}(0)=0, c_{2} \leq 0$. Hence and by 4.2 we have that

$$
0 \geq c_{2} \geq \mathcal{J}(v)-\varepsilon
$$

If we assume that $v \in \partial \Omega$, then $\mathcal{J}(v)>0$, by Step 2 in the proof of Theorem 3.1. Taking sufficiently small $\varepsilon$, we deduce that $0 \geq c_{2} \geq \mathcal{J}(v)-\varepsilon>0$, which is a contradiction.

Take $w=v+s h$ with $0<s \leq 1, h \in \mathbf{W}^{1} \mathbf{L}^{G}$ such that $\|h\|_{\mathbf{W}^{1} \mathbf{L}^{G}}=1$. Then, by Lemma 2.2 we have that

$$
\int_{I} G(v+s h) d t \leq \int_{I} G(v)+\sqrt{s}|G(2 v)-2 G(v)|+2 G(\sqrt{s} h) d t
$$


and

$$
\int_{I} G(\dot{v}+s \dot{h}) d t \leq \int_{I} G(\dot{v})+\sqrt{s}|G(2 \dot{v})-2 G(\dot{v})|+2 G(\sqrt{s} \dot{h}) d t
$$

Hence

$$
\begin{aligned}
\Phi(v+s h) \leq & \int_{I} G(v)+G(\dot{v})+\sqrt{s}(|G(2 v)-2 G(v)|+|G(2 \dot{v})-2 G(\dot{v})|) d t \\
& +2 \int_{I} G(\sqrt{s} h)+G(\sqrt{s} \dot{h}) d t .
\end{aligned}
$$

Note that $\|\sqrt{s} h\|_{\mathbf{L}^{G}} \leq 1$. From $(2.1)$ we obtain $\int_{I} G(\sqrt{s} h) \leq\|\sqrt{s} h\|_{\mathbf{L}^{G}} \leq \sqrt{s}$. Hence

$$
\Phi(v+s h) \leq \int_{I} G(v)+G(\dot{v})+\sqrt{s}(|G(2 v)-2 G(v)|+|G(2 \dot{v})-2 G(\dot{v})|) d t+4 \sqrt{s} .
$$

Since $\Phi(v)<\rho$, it follows that for $s$ sufficiently small $\Phi(v+s h)<\rho$. By 4.4),

$$
\mathcal{J}(v+s h) \geq \mathcal{J}(v)-\sqrt{\varepsilon}\|s h\|_{\mathbf{W}^{1} \mathbf{L}^{G}}
$$

Hence

$$
\frac{\mathcal{J}(v+s h)-\mathcal{J}(v)}{s} \geq-\sqrt{\varepsilon}
$$

Taking the limit as $s \rightarrow 0$, we have $\left\langle\mathcal{J}^{\prime}(v), h\right\rangle \geq-\sqrt{\varepsilon}$ for $h \in \mathbf{W}^{1} \mathbf{L}^{G}$ such that $\|h\|_{\mathbf{W}^{1} \mathbf{L}^{G}}=$ 1. Since $-h \in \Omega$, we have $\sup _{\|h\|=1}\left|\left\langle\mathcal{J}^{\prime}(v), h\right\rangle\right| \leq \sqrt{\varepsilon}$ and hence

$$
\left\|\mathcal{J}^{\prime}(v)\right\|_{\left(\mathbf{W}^{1} \mathbf{L}^{G}\right)^{\star}} \leq \sqrt{\varepsilon} .
$$

Let $\left\{u_{n}\right\}$ be a minimizing sequence of $\mathcal{J}$. We choose $\varepsilon_{n}$ in the following way

$$
\varepsilon_{n}= \begin{cases}\mathcal{J}\left(u_{n}\right)-\inf _{\bar{\Omega}} \mathcal{J}, & \mathcal{J}\left(u_{n}\right)>\inf _{\bar{\Omega}} \mathcal{J} \\ \frac{1}{n}, & \mathcal{J}\left(u_{n}\right)=\inf _{\bar{\Omega}} \mathcal{J}\end{cases}
$$

One can see, that $\varepsilon_{n} \rightarrow 0$ as $n \rightarrow \infty$. Since $u_{n}$ satisfies 4.1 for each $n$, we have

$$
c_{2} \leq \mathcal{J}\left(v_{n}\right) \leq c_{2}+\varepsilon_{n}, \quad\left\|v_{n}-u_{n}\right\|_{\mathbf{W}^{1} \mathbf{L}^{G}} \leq \sqrt{\varepsilon_{n}}, \quad\left\|\mathcal{J}^{\prime}\left(v_{n}\right)\right\|_{\left(\mathbf{W}^{1} \mathbf{L}^{G}\right)^{\star}} \leq \sqrt{\varepsilon_{n}}
$$

for $v_{n}$ associated to $u_{n}$ and $\varepsilon_{n}$ in 4.2 -4.4. Hence we can see that $\left\{v_{n}\right\}$ is a PalaisSmale sequence of $\mathcal{J}$. Since $\mathcal{J}$ satisfies the Palais-Smale condition (Step 2 in the proof of Theorem 3.1), we have that there is $u_{2}$ such that $\mathcal{J}\left(u_{2}\right)=c_{2}$ and $\mathcal{J}^{\prime}\left(u_{2}\right)=0$, so $u_{2}$ is the desired solution of (1.1).

Remark 4.3. Similar arguments regarding the existence of a second solution can be found in [13, Proof of Lemma 3.3] and [21, Step 4 in the proof of Theorem 1.1]. See also [16, Theorem 4.2, Corollary 4.1] and [3, Theorem 3.1] for more detailed computations. 
If $f(t) \equiv 0$ it is necessary to show that $\inf _{u \in \bar{\Omega}} \mathcal{J}(u)<0$. Without this assumption it is possible that the minimizing sequence found in the proof of Theorem 4.2 converges to the solution $u_{0} \equiv 0$. In order to avoid this phenomenon we add Assumptions $\left(\mathrm{F}_{6}\right)$ and $\left(\mathrm{V}_{5}\right)$.

Theorem 4.4. Let $F$ and $V$ satisfies $\left(\mathrm{F}_{1}\right)-\left(\mathrm{F}_{6}\right),\left(\mathrm{V}_{1}\right)-\left(\mathrm{V}_{5}\right)$ and $f(t) \equiv 0$. Then (1.1) has at least two nontrivial periodic solutions.

Proof. Let $0 \neq \psi \in \Omega$. Then, by Lemma 2.1 . $G\left(\frac{\psi(t)}{2|I|}\right) \leq \rho / 2$ for all $t \in I$. Choose

$$
\lambda<\min \left\{1,\left(\frac{C_{W}}{C_{F K}}\right)^{1 /\left(\min \left\{\zeta_{F}, \zeta_{K}\right\}-\zeta_{W}\right)}\right\} \text {, }
$$

where $C_{W}=\int_{I} \bar{W}(t, \psi) d t, C_{F K}=\int_{I} F(t, \psi, \dot{\psi})+K(t, \psi) d t$.

By $\left(\mathrm{F}_{6}\right),\left(\mathrm{V}_{5}\right)$ and $f(t) \equiv 0$ we have

$$
\begin{aligned}
\mathcal{J}(\lambda \psi) & =\int_{I} F(t, \lambda \psi, \lambda \dot{\psi})+V(t, \lambda \psi) d t \\
& \leq \int_{I} \lambda^{\zeta_{F}} F(t, \psi, \dot{\psi})+\lambda^{\zeta_{K}} K(t, \psi)-\lambda^{\zeta_{W}} \bar{W}(t, \psi) d t \\
& \leq C_{F K} \lambda^{\min \left\{\zeta_{F}, \zeta_{K}\right\}}-C_{W} \lambda^{\zeta_{W}}<0 .
\end{aligned}
$$

Hence $c_{2}<0$. In the same way as in the proof of Theorem 4.2 we show that there exists a minimizing sequence $\left\{v_{n}\right\}$ such that $\mathcal{J}\left(v_{n}\right) \rightarrow 0$ and $\mathcal{J}^{\prime}\left(v_{n}\right) \rightarrow c_{2}$ as $n \rightarrow \infty$. Hence there is $u_{2}$ such that $\mathcal{J}\left(u_{2}\right)=c_{2}$ and $\mathcal{J}^{\prime}\left(u_{2}\right)=0$ and $u_{2}$ is the second solution of (1.1). Since $c_{2}<0$ and $\mathcal{J}(0)=0, u_{2}$ is nontrivial.

Remark 4.5. Theorem 4.4 is some generalization of existing results for (see for example $13,22]$ ). Although we consider only periodic problem, but we do it for more general kinetic part of Lagrangian.

We can consider simpler class of potentials

$$
V(t, x)=A_{0}(t) G_{0}(x)-A_{1}(t) G_{1}(x)-A_{2}(t) G_{2}(x)
$$

where $G_{i}: \mathbb{R}^{N} \rightarrow[0, \infty)$ are convex functions of class $C^{1}$ satisfying $G_{i}(0)=0$ (to provide $\left.\left(\mathrm{V}_{4}\right)\right)$ and assumptions $\Delta_{2} \nabla_{2}$ in infinity with Simonenko indices (see $\left.|7|\right) \min \left\{p_{G_{1}}^{\infty}, p_{G_{2}}^{\infty}\right\}>$ $q_{G_{0}}^{\infty}\left(\right.$ to provide $\left.\left(\mathrm{V}_{2}\right)\right)$. To provide $\left(\mathrm{V}_{3}\right)$ we assume that $A_{1}(t) G_{1}(x)<g(t)$ for $G(x /(2|I|))<$ $\rho / 2, A_{0}(t)>b$ and

$$
\lim _{G(x /(2|I|)) \rightarrow 0} \frac{G_{2}(x)}{G(x)}=0 .
$$

To ensure that Assumption $\left(\mathrm{V}_{5}\right)$ is satisfied we need that $G_{1}$ satisfies $\Delta_{2}$ globally and $G_{0}$ satisfies $\nabla_{2}$ globally with Simonenko indices $q_{G_{1}}<p_{G_{0}}$. 


\section{Example}

We finish the paper with some example of function $F$, potential and forcing satisfying Assumptions $\left(\mathrm{V}_{1}\right)-\left(\mathrm{V}_{5}\right),\left(\mathrm{F}_{1}\right)-\left(\mathrm{F}_{6}\right)$ and $(\mathrm{f})$. We also show that they do not satisfy assumptions in 7, which indicate that taking $\Phi^{-1}([0, \rho))$ instead of the ball $\left\{u \in \mathbf{W}^{1} \mathbf{L}^{G}\right.$ : $\left.\|u\|_{\mathbf{W}^{1} \mathbf{L}^{G}}<r\right\}$ is better to obtain the mountain pass geometry in the anisotropic case.

Let us recall that in [7] condition (1.3), mentioned in the introduction, was guaranteed by the following assumptions:

$\left(\mathrm{A}_{3}\right)$ there exist $r_{0}, b>1$ and $g \in \mathbf{L}^{1}(I, \mathbb{R})$, such that $V(t, x) \geq b G(x)-g(t)$ for $|x| \leq r_{0}$, either

$$
\int_{-T}^{T} G^{*}(f(t))+g(t) d t<\min \{1, b-1\}\left(r_{0} /\left(2 C_{\infty, G}\right)\right), \quad r_{0} \geq 2 C_{\infty, G}
$$

or

$$
\int_{-T}^{T} G^{*}(f(t))+g(t) d t<\min \{1, b-1\} \begin{cases}\left(r_{0} /\left(2 C_{\infty}, G\right)\right)^{q_{G}}, & r_{0} \leq 2 C_{\infty, G} \\ \left(r_{0} /\left(2 C_{\infty}, G\right)\right)^{p_{G},}, & r_{0}>2 C_{\infty, G}\end{cases}
$$

where $C_{\infty, G}$ is an embedding constant for $\mathbf{W}^{1} \mathbf{L}^{G} \hookrightarrow \mathbf{L}^{\infty}$ given by formula $C_{\infty, G}=$ $\max \{1,|I|\} A_{G}^{-1}\left(\frac{1}{|I|}\right), A_{G}:[0, \infty) \rightarrow[0, \infty)$ is the greatest convex minorant of $G$ (see $\left.17 \mid\right)$, $r=r_{0} / C_{\infty, G}$.

Sets of assumptions

(i) $\left(\mathrm{A}_{3}\right),(5.1)$ or 5.2$)$,

(ii) $\left(\mathrm{V}_{3}\right),(\mathrm{f})$

are independent, namely for some potentials it is not possible to find $r_{0}$ such that the first assumptions are satisfied, but for the same potential one can find $\rho$ such that the latter are met.

Example 5.1. Let $I=[-1,1], x=\left(x_{1}, x_{2}\right), v=\left(v_{1}, v_{2}\right) \in \mathbb{R}^{n} \times \mathbb{R}^{n}$,

$$
\begin{gathered}
F(t, x, v)=G(v)=v_{1}^{2}+\left(v_{1}-v_{2}\right)^{4}, \quad K(t, x)=2 G(x)+|x|^{2} \log \left(|x|^{2}+1\right), \\
W(t, x)=(G(x))^{2}+\frac{|x|^{3 / 2}+|x|^{5}}{100}, \quad V(t, x)=K(t, x)-W(t, x), \\
f_{0}(t)=\frac{2-t^{2}}{250}, \quad f=\left(f_{0}, \ldots, f_{0}\right), \quad g(t) \equiv 0.001, \quad b=2, \quad \rho=0.004, \\
\theta_{V}=4.9, \quad \varepsilon_{V}=0.001, \quad \theta_{F}=4, \quad \zeta_{W}=\frac{31}{16}, \quad \zeta_{K}=2, \quad \zeta_{F}=2 .
\end{gathered}
$$


If we take $F, V$ and $f$ given above one can check, that $\left(\mathrm{V}_{1}\right)-\left(\mathrm{V}_{5}\right),\left(\mathrm{F}_{1}\right)-\left(\mathrm{F}_{6}\right)$ and (f) are satisfied. However, there do not exist $a, b, r_{0}$ such that either $\left(\mathrm{A}_{3}\right)$ and (5.1) or $\left(\mathrm{A}_{3}\right)$ and (5.2) holds. This situation was shown in Figure 5.1

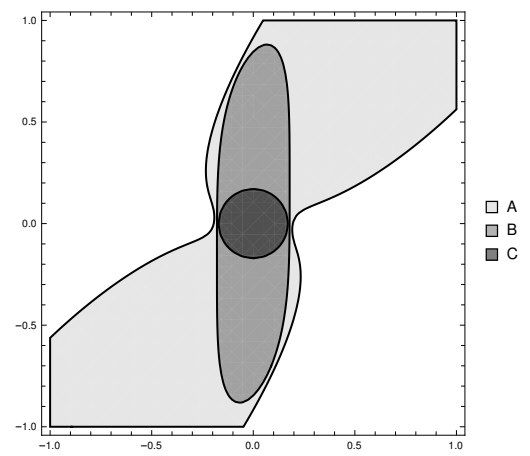

Figure 5.1: The shape of $A$ is such that for a ball $B=B_{r_{0}}(0)$ with $r_{0}$ such that $B \subset A$ neither (5.1) nor (5.2) is satisfied. For $C \subset A$ condition (f) is satisfied.

The shape of the area

$$
A=\left\{x \in \mathbb{R}^{n} \times \mathbb{R}^{n}: V(x) \geq b G(x)-g\right\}
$$

is such that for a ball

$$
B=\left\{x \in \mathbb{R}^{n} \times \mathbb{R}^{n}:|x| \leq r_{0}\right\}
$$

with sufficiently small radius (such that $B \subset A$ ), neither (5.1) nor (5.2) is satisfied. If, instead of the ball, we take "more anisotropic" area

$$
C=\left\{x \in \mathbb{R}^{n} \times \mathbb{R}^{n}: G\left(\frac{x}{2|I|}\right) \leq \frac{\rho}{2}\right\} \subset A,
$$

connected with condition $\left(\mathrm{V}_{3}\right)$, then (f) is satisfied.

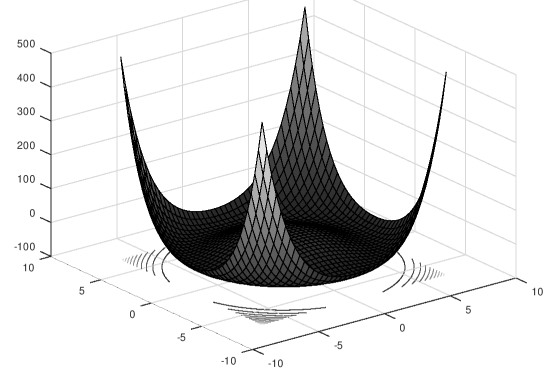

Figure 5.2: Plot of the function $h_{1}$.

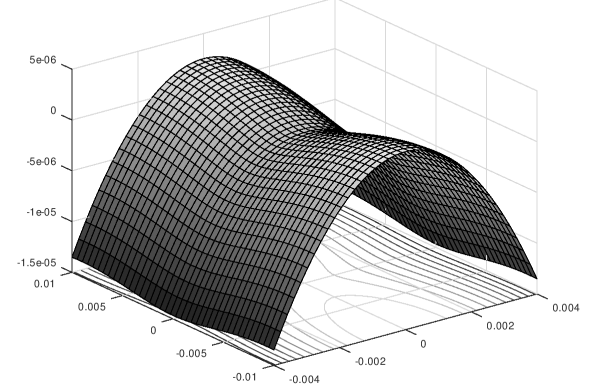

Figure 5.3: Plot of the function $h_{2}$. 
- If we assume that (5.1) is met, then for each $r_{0}>2 C_{G, \infty}$ there exists $x \in B_{r_{0}}(0)$ such that $V(x)<b G(x)-g$ for all $b>1, g<\frac{r_{0}}{2 C_{\infty, G}}$, which contradicts assumption $\left(A_{3}\right)$.

In fact, taking $x \in \partial B_{r_{0}}(0)$ it suffices to show that function $h_{1}: \mathbb{R}^{n} \times \mathbb{R}^{n} \rightarrow \mathbb{R}$, $h_{1}(x)=G(x)-V(x)-\frac{|x|}{2 C_{G, \infty}}$ can have positive values for any $r_{0}>2 C_{G, \infty}$ (see Figure 5.2.

- If we assume that $(5.2)$ is met, then for each $r_{0}>0$ there exists $x \in B_{r_{0}}(0)$ such that $V(x)<b G(x)-g$ for all $b>1, g<\min \left\{\left(\frac{r_{0}}{2 C_{\infty, G}}\right)^{2},\left(\frac{r_{0}}{2 C_{\infty, G}}\right)^{4}\right\}$.

In fact, taking $x \in \partial B_{r_{0}}(0)$ it suffices to show that function $h_{2}: \mathbb{R}^{n} \times \mathbb{R}^{n} \rightarrow \mathbb{R}$, $h_{2}(x)=G(x)-V(x)-\left(\frac{|x|}{2 C_{G, \infty}}\right)^{2}$ can have positive values for any $r_{0}$ (see Figure 5.3 .

Remark 5.2. In the above example we can also take a more complicated $F$, e.g.,

$$
F(t, x, v)=G(v)\left(2+|x|^{9 / 2}-\sin t\right)
$$

\section{Acknowledgments}

I would like to thank the referee for valuable comments and suggestions.

\section{References}

[1] S. Acinas, L. Buri, G. Giubergia, F. Mazzone and E. Schwindt, Some existence results on periodic solutions of Euler-Lagrange equations in an Orlicz-Sobolev space setting, Nonlinear Anal. 125 (2015), 681-698.

[2] A. Ambrosetti and P. H. Rabinowitz, Dual variational methods in critical point theory and applications, J. Functional Analysis 14 (1973), no. 4, 349-381.

[3] V. Ambrosio and H. Hajaiej, Multiple solutions for a class of nonhomogeneous fractional Schrödinger equations in $\mathbb{R}^{N}$, J. Dynam. Differential Equations 30 (2018), no. $3,1119-1143$.

[4] G. Barletta and A. Cianchi, Dirichlet problems for fully anisotropic elliptic equations, Proc. Roy. Soc. Edinburgh Sect. A 147 (2017), no. 1, 25-60.

[5] H. Brézis and E. Lieb, A relation between pointwise convergence of functions and convergence of functionals, Proc. Amer. Math. Soc. 88 (1983), no. 3, 486-490.

[6] M. Chmara and J. Maksymiuk, Anisotropic Orlicz-Sobolev spaces of vector valued functions and Lagrange equations, J. Math. Anal. Appl. 456 (2017), no. 1, 457-475. 
[7] _ Mountain pass type periodic solutions for Euler-Lagrange equations in anisotropic Orlicz-Sobolev space, J. Math. Anal. Appl. 470 (2019), no. 1, 584-598.

[8] _ Mountain pass solutions to Euler-Lagrange equations with general anisotropic operator, J. Math. Anal. Appl. 485 (2020), no. 2, 123809, 14 pp.

[9] A. Daouas, Existence of homoclinic orbits for unbounded time-dependent p-Laplacian systems, Electron. J. Qual. Theory Differ. Equ. 2016 (2016), Paper No. 88, 12 pp.

[10] I. Ekeland, On the variational principle, J. Math. Anal. Appl. 47 (1974), 324-353.

[11] P. Harjulehto and P. Hästö, Orlicz spaces and generalized Orlicz spaces, Lecture Notes in Mathematics 2236, Springer, Cham, 2019.

[12] Y. Jabri, The Mountain Pass Theorem: Variants, generalizations and some applications, Encyclopedia of Mathematics and its Applications 95, Cambridge University Press, Cambridge, 2003.

[13] J. Janczewska, Two almost homoclinic solutions for second-order perturbed Hamiltonian systems, Commun. Contemp. Math. 14 (2012), no. 4, 1250025, 9 pp.

[14] M. A. Khamsi and W. M. Kozlowski, Fixed Point Theory in Modular Function Spaces, Birkhäuser/Springer, Cham, 2015.

[15] V. K. Le, On second order elliptic equations and variational inequalities with anisotropic principal operators, Topol. Methods Nonlinear Anal. 44 (2014), no. 1, $41-72$.

[16] J. Mawhin and M. Willem, Critical Point Theory and Hamiltonian Systems, Applied Mathematical Sciences 74, Springer-Verlag, New York, 1989.

[17] F. D. Mazzone and S. Acinas, Periodic solutions of Euler-Lagrange equations in an anisotropic Orlicz-Sobolev space setting, Rev. Un. Mat. Argentina 60 (2019), no. 2, no. $2,323-341$.

[18] J. Musielak, Orlicz Spaces and Modular Spaces, Lecture Notes in Mathematics 1034, Springer-Verlag, Berlin, 1983.

[19] G. Schappacher, A notion of Orlicz spaces for vector valued functions, Appl. Math. 50 (2005), no. 4, 355-386.

[20] S. Tersian, On symmetric positive homoclinic solutions of semilinear p-Laplacian differential equations, Bound. Value Probl. 2012 (2012), no. 121, 14 pp. 
[21] Z. Zhang and R. Yuan, Existence of two almost homoclinic solutions for $p(t)$ Laplacian Hamiltonian systems with a small perturbation, J. Appl. Math. Comput. 52 (2016), no. 1-2, 173-189.

[22] _ Homoclinic solutions for p-Laplacian Hamiltonian systems with combined nonlinearities, Qual. Theory Dyn. Syst. 16 (2017), no. 3, 761-774.

Magdalena Chmara

Department of Technical Physics and Applied Mathematics, Gdańsk University of Technology, Narutowicza 11/12, 80-233 Gdańsk, Poland

E-mail address: magdalena.chmara@pg.edu.pl 